\title{
Developing A 3D Printable Electret Material For Sensing Applications.
}

\author{
O. A. Omoniyi, B. Tiller, R. O’Leary, J. F.C. Windmill \\ Centre for Ultrasonic Engineering, Electronic \& Electrical Engineering Dept., \\ Bioacoustics Group, University of Strathclyde \\ Glasgow, Scotland, United Kingdom \\ Oluwaseun.omoniyi@strath.ac.uk
}

\section{SUMMARY AND MOTIVATION}

The introduction of three-dimensional (3D) printing technology has opened up a world of rapid product development in different fields. 3D printing of piezoelectric materials has been growing in popularity in recent years showing increasing promise in the design of miniature sensors for acoustic devices, diagnostics devices, and energy harvesters. In this study, we demonstrate that voided polymer electret material can be printed into 3D structures using Stereolithography (SL) printing technology. A test sample was fabricated by printing a thin membrane of voided polymer electret sandwiched between two base structures. The polymer electret is formed by incorporating dry expanded Expancel microspheres into a photoactive polymer solution of polyethylene glycol diacrylate (PEGDA). The average $d_{33}$ coefficient of the material were determined as $58 \mathrm{pm} / \mathrm{V}$. The results obtained showed the potential of using a voided polymer composite for 3D-printing functional electret based sensing devices.

\section{ADVANCES OVER PREVIOUS WORKS}

The piezoelectric properties of certain polymers have been studied in depth and much work has been done to exploit this advantage [1-2]. Polymers such as polytetrafluoroethylene (PTFE), polyvinylidenefluoride (PVDF), and Polypropylene (PP) have been shown to possess good piezoelectric properties and enhanced charge retention [3]. However, it has been shown that a new class of polymer electrets, the voided cellular polymer electrets, offers a number of advantages [6]. An electret is a dielectric material with quasi - permanent polarization. Voided polymer electrets are polymer materials consisting of internal voids, which are electrically charged. These materials have been reported to have higher piezoelectric constants due to their soft internal structure, when compared to other piezoelectric polymers, and offer better acoustic impedance matching in water and air coupled ultrasonic applications [4-5].

The possibility of fabricating miniature devices with enhanced operational capabilities and complex geometries with reduced cost using piezocomposites has been shown in [1].

In this study, we show that a sensing device can fabricated through a combination of 3D printing with cellular polymer electret technology

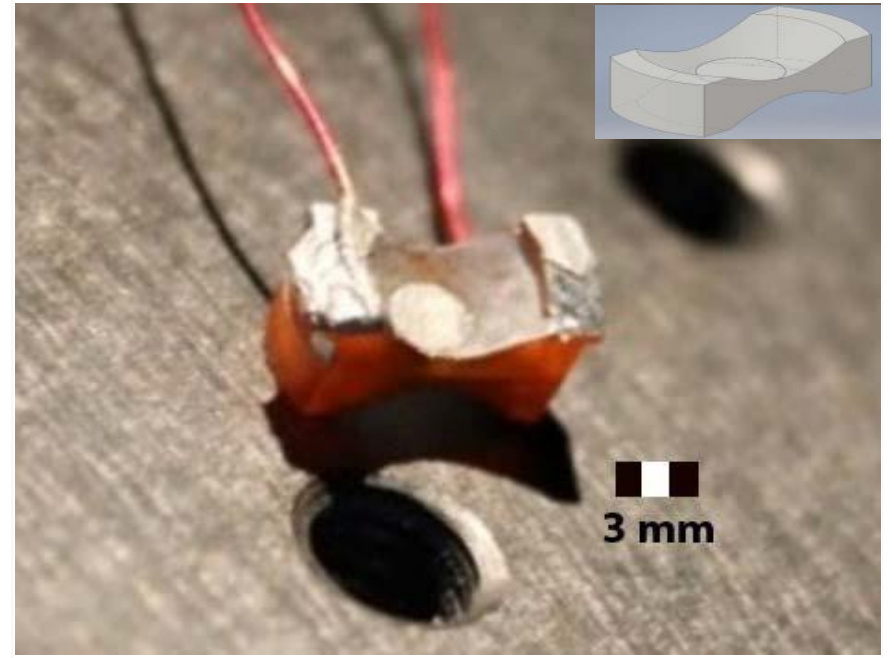

Fig. 1: Shows the 3D printed device (top) and its CAD model. The electret membrane is scaled to size.

\section{MATERIALS AND METHODS}

The materials used in this experiment include commercially available Expancel microspheres DE with particle size $30 \mu \mathrm{m}$ (Akzo Noble pulp, Sundsvall Sweden), polyethylene glycol diacrylate (PEGDA), phenylbis (2,4,6-trimethylbenzoyl), phosphine oxide (Irgacure 819), and Sudan I (Sigma Aldrich).

\section{A. Material Synthesis and $3 D$ printing}

The material synthesis was carried out in two steps. Two different polymer-based materials were synthesized to 3D-print the sample reported in this work. The first step consisted of mixing PEGDA with Irgacure 819 at $1 \mathrm{wt} \%$. Irgacure 819 is a photoinitiator, necessary to start the polymerization process of PEGDA when exposed to ultraviolet (UV) light. The mixture was stirred using a magnetic mixer for 24 hours to ensure a homogenous mix. After the mixing process, $0.1 \mathrm{wt} \%$ of SUDAN I was added into the mixture. SUDAN I is a UV blocker that allows an improvement of the resolution of the 3D-printed part. To ensure a homogenous composition, the mixture was put in a THINKY AER-250 mechanical mixer, mixing the composite for 3 minutes at $1500 \mathrm{rpm}$, and defoaming it for 2 minutes at $1200 \mathrm{rpm}$. The second step 
involved mixing Expancel microspheres $(\mathrm{EP} \mu \mathrm{S})$, with PEGDA at a ratio of 1 to 15 . Thus, 4 grams of $\mathrm{EP} \mu \mathrm{S}$ were added into 60 grams of PEGDA, and the mixture was mechanically mixed following the aforementioned process. After mechanical mixing a control PEGDA:EP $\mu \mathrm{S}$ sample was made using a screen printing technique, with a layer thickness of $80 \mu \mathrm{m}$, using an RK control coater. Every layer was cured under UV light for two minutes using an Intertronics IUV250 Hand Lamp (Intertronics, Kidlington, England, UK).

An ASIGA PicoPlus27 3D printer (ASIGA, Anaheim Hills, California, USA) was used to print the device using the materials created in both steps above. Both of them were 3Dprinted at a slice thickness resolution of $10 \mu \mathrm{m}, 5 \mathrm{sec}$ exposure time and $100 \mathrm{sec}$ burn in time. The support structure was 3Dprinted first using the initial mixture of only PEGDA. By pausing the 3D printing process at the correct build layer, it was possible to switch the build fluid to print a membrane of PEGDA: $\mathrm{EP} \mu \mathrm{S}$ of $0.21 \mathrm{~mm}$. By pausing the 3D printing process at the correct build layer, it was possible to switch again to the PEGDA fluid and print the second part of the support structure. The final membrane consisted of a disk of $0.21 \mathrm{~mm}$ thickness and a circle of $3 \mathrm{~mm}$ in diameter as shown in Fig. 1. After 3D-printing, Thin film coating of the sample was done using silver as the contact electrode. The membrane was poled using the corona discharge technique, which involved charging the sample under a pin electrode at a distance of $2 \mathrm{~cm}$ by applying a constant voltage of positive polarity at $12 \mathrm{kV}$. This process was carried out for $60 \mathrm{mins}$.

\section{EXPERIMENTAL RESULTS}

\section{A. Structural Analysis}

The microstructure of electret membrane was investigated using a table-top scanning electron microscope at an accelerating voltage of $15 \mathrm{kV}$ (SEM, Hitachi TM1000, Krefeld, Germany). The microstructure of the electret was investigated to determine the degree of bonding between the voids and matrix and the dispersion of the voids since this greatly affects the poling result and thus piezoelectric and mechanical properties of composites [10].

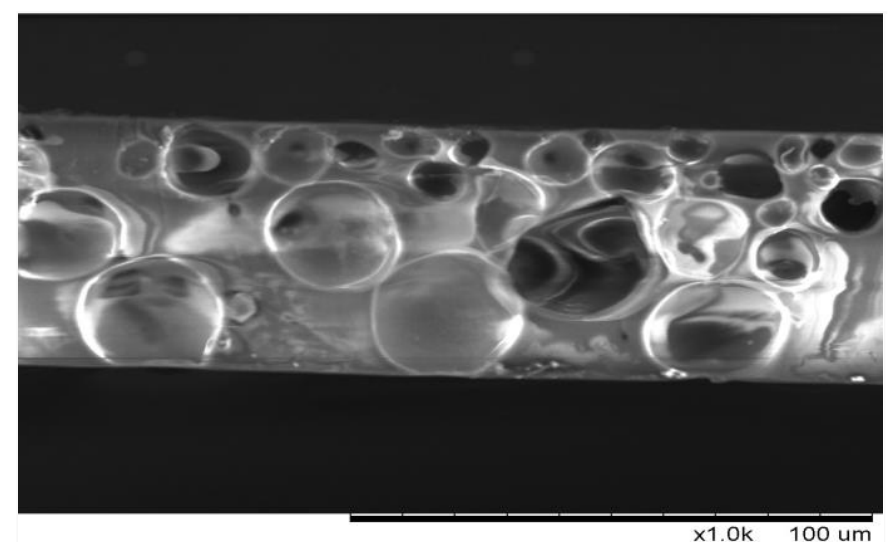

Fig. 2: Shows the cross-section of the device membrane. The internal voids are clearly visible and appears smooth and homogenous. Although voids sizes of $60 \mu \mathrm{m}$ were used, smaller sized voids are clearly seen. Spaces observed in the micrographs appear to be as a result of the mixing technique used.

\section{B. Piezoelectric properties}

The piezoelectric properties of the device were investigated using a microscanning laser Doppler vibrometer (Polytec MSA100-3D, Waldbronn, Germany) technique [11]. The voltage stimulus was generated by the MSA100-3D internal data acquisition board. The analysis of the device's velocity and displacement was determined with the laser vibrometer's control PC and data management system. A sinusoidal AC voltage of $3 \mathrm{~V}_{\mathrm{pp}}$ was applied across the axis of charging and the resulting displacement along the poling axis was measured. A frequency sweep stimulus was applied from $100 \mathrm{~Hz}$ to $25 \mathrm{kHz}$ in order to obtain the characteristic frequencies at which the device will respond, as shown in Fig. 3. The average $d_{33}$ coefficient of the material was determined as $58 \mathrm{pm} / \mathrm{V}$.
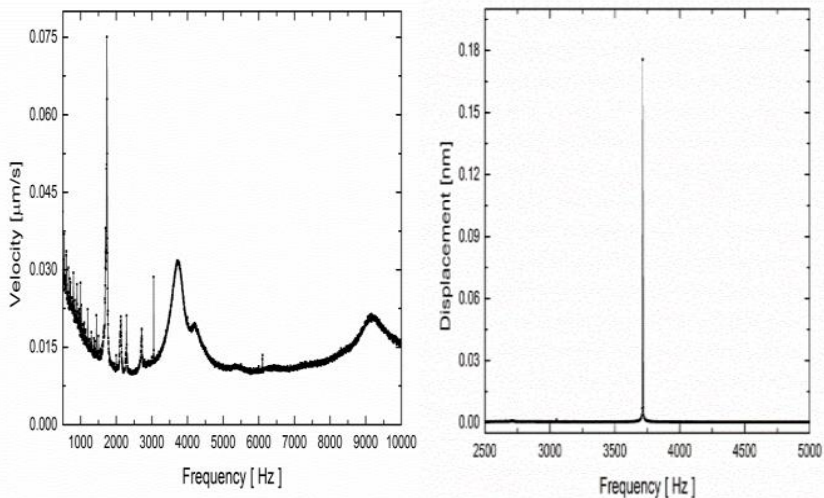

Fig. 3. Shows the response of the 3D printed membrane to a frequency sweep, and the magnitude of mechanical displacement (peak to peak) in response to a sinusoidal voltage of $3 \mathrm{~V}_{\mathrm{pp}}$, at a frequency of $3.7 \mathrm{kHz}$.

\section{CONCLUSION}

In conclusion this work demonstrates the possibility to 3Dprint a cellular polymer electret. The 3D-printing process employed allowed the fabrication of a device with different material layers, and good resolution. The device consists of a 3D-printed voided polymer electret thin membrane sandwiched between a solid base of 3D-printed polymer. From the analysis obtained, it was shown that the voided polymer electret membrane is piezoelectric and can be employed in the fabrication of MEMS devices. Furthermore, the device provided a good response at a broader range of frequencies. This work also gives an insight into the creation of functional 3D-printed sensors through a cheaper and more time-efficient process.

Further investigation is still required to verify the volume of the voids and the charge distribution during poling as these are assumed to play a very important role in the electromechanical properties of the new electret material. Mechanical properties and Finite element modelling of the material would also be carried out to understand the physics of the material and its properties. 


\section{REFERENCES}

[1] K. Kim, W.Zhu, X. Qu, C Aaronson, W. R. McCall, S. Chen, D. J. Sirburly., "3D Optical Printing of Piezoelectric Nanoparticle - Polymer Composite Materials", ACS Nano., vol. 8 (10), pp. 9799-9806, 2014.

[2] C. Brown, R. Kell, R. Taylor and L. Thomas, "Piezoelectric Materials, A Review of Progress", IRE Transaction on Component Parts, vol. 9 (7), pp. 193-211, 1962.

[3] J. Windmill, A Zorab, D. Bedwell, D. Robert, "Nanomechanical and electrical characterization of a new cellular electret sensor-actuator", Nanotechnology., vol. 035506 (7pp), 2008.

[4] R. Kressmann, "New piezoelectric polymer for air-borne and water borne sound transducers", Journal Acoustic Society of America, vol. 109(4), pp. 1412-1416, 2001.

[5] V. Bovtun, J. Doring, J. Bartusch, U. Beck, A. Erhard, Y. Yakymento, "Air-Coupled Ultrasonic Transducers Based on Cellular Polypropylene Ferroelectret Films", Ferroelectrics., vol. 0193, pp. 186-192, 2007.

[6] M. Wegener and S. Bauer, "Microstorms in cellular polymers: a route to soft piezoelectric transducer materials with engineered macroscopic dipoles", Chemphyschem:a European journal of chemical physics and physical chemistry, vol. 6 (6), pp. 1014-1025, 2005.

[7] S.Bauer, "Piezo-, pyro- and ferroelectrets: soft transducer materials for electromechanical energy conversion", IEEE Trans. Dielectr. Electr. Insul., vol. 13, pp. 95362, 2006.

[8] M. Paajanen, J. Lekkala, K. Kirjavainen, "Electromechanical film (EMFi) - a new multipurpose electret material”, Elsevier., vol. 84 (7), pp. 95-102, 2000.

[9] S. Rajala, J. Lekkala, "Pvdf and emfi sensor materials - A comparative study", Proc. eurosensors, vol. 5, pp. 862-865, 2010.

[10] R. Y. Ting, Composite Piezoelectric Materials for Transduction, Appl. Acoust., vol 41, pp. 325-335, 1994

[11] Yao, K., \& Tay, F. E. H. Measurement of longitudinal piezoelectric coefficient of thin films by a laser-scanning vibrometer. IEEE Transactions on Ultrasonics, Ferroelectrics, and Frequency Control. https://doi.org/10.1109/TUFFC.2003.1182115 